\title{
t-words: Playing with Sounds and Creating Narratives
}

\author{
Cristina Sylla $^{1}$, Sérgio Gonçalves ${ }^{1}$, Pedro Branco ${ }^{2}$, and Clara Coutinho ${ }^{1}$ \\ ${ }^{1}$ Engagelab/CIEd, University of Minho, Braga/Guimarães, Portugal \\ \{sylla, sgoncalves\} @engagelab.org, ccoutinho@ie.uminho.pt \\ ${ }^{2}$ EngageLab/Dep. of Information Systems, University of Minho, Guimarães, Portugal \\ pbrancoedsi.uminho.pt
}

\begin{abstract}
We present $\mathrm{t}$-words an interface for children to playful explore sounds, words and sentences while developing pre-literate skills. The interface consists of rectangular blocks in which children can record and then play the recorded audio. Additionally children can personalize the blocks by drawing on their surface. Children can engage in different literacy related activities such as building rhymes, playing with sounds and words as well as trying out different combinations of sentences while engaging in storytelling. Since the interface targets audio skills it may foster the development of phonological awareness and sensitiveness, helping to promote children's early literacy.
\end{abstract}

Keywords: Tangible Interfaces, Story Listening System, Storytelling, Children, Emergent Literacy, Phonological Awareness.

\section{Introduction}

Emergent literacy, a term first coined by Clay [3] sees reading and writing as a continuous process that begins in the early years of childhood, through children's exposure to environments where literacy is present. This means that children have contact with books, for instance through shared reading, listening to and telling stories, or by engaging in language games. Emergent literacy consists of the skills that lead to the development of reading and writing. From this perspective, reading, writing and language development are developmental processes that are intertwined with each other. Inside-out and inside-in skills [7] are two domains of emergent literacy. Inside-out skills refer to phonological awareness, and letter knowledge, it presupposes the ability to map letters to sounds and sounds to words. Outside-in skills refer to the knowledge of the narrative's conceptual and semantic context, which implies knowing the words that are used and the context in which the narrative takes place. Studies suggest that reading in the 1st and 2 nd grades is strongly determined by the inside-out skills that children have acquired at the end of pre-school [7].

\section{Background}

The idea behind t-words was to develop an interface where children can playful engage with sounds and words, that can be used to deepen young children's 
phonological awareness and sensitivity, promoting the acquisition of new vocabulary and ultimately develop their early literacy skills. Additionally the interface should encourage collaborative work and team spirit, as well as promote exchange of experiences and knowledge. There are numerous systems where children can play with language elements, while engaging as story authors $[1,4,5,6]$. $t$-words positions itself in between TellTale [1] and the Siftables [4]: each piece can record audio and be written and drawn on the surface and all the pieces can be connected together to playback the recorded sounds, furthermore t-words has no screen or need of a computer, thus facilitating digital inclusion in contexts with less technological infrastructures.

\section{The Interface}

The t-words interface comprises a set of different sized blocks capable of recording and playing sounds, words or sentences. The blocks can either have the same or different sizes. Children can customize the blocks with their drawings, which can be drawn on top of the blocks' surface. Each block has a recorder embedded except the player-block, which has a player instead (Fig.1). Every block has a button to start and stop recording and a light bar that gives the user information about the recording and playback sound level.

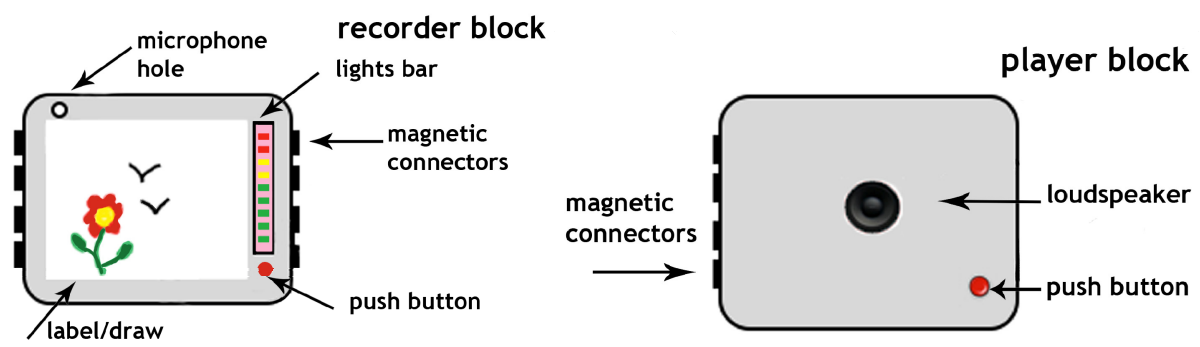

Fig. 1. Recorder-block (left), player-block (right)

The player-block has a loudspeaker, and an electronic system that gathers information from the recorder-blocks. The communication between the blocks happens through magnetic connectors, which allows building a well-aligned sequence of recorder-blocks and player and simultaneous permits the electrical communication between them (sending/receiving data signals as well as power supply). Children can record audio in one or more blocks depending on the proposed activities. To play the recorded audio children snap the player-block to the block or block sequence in which they have recorded the audio. In case the audio is recorded in more than one block, the recorded blocks have to be snapped together with the player-block at the end (Fig.2). 


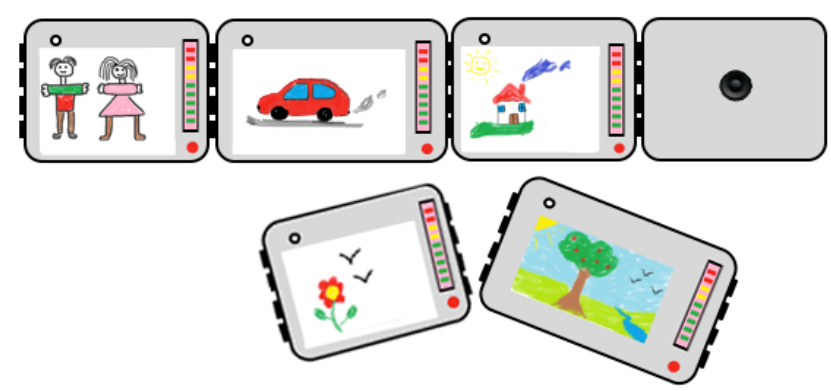

Fig. 2. Sequence with recorder-blocks and a player-block

\subsection{Technical Description}

The recorder has an embedded microphone, which is connected to a microcontroller through an analog-to-digital converter (Fig.3 left). The microcontroller is an Arduino [2] compatible very small and low power microprocessor. A flash memory, which is connected to the microcontroller, is used to store the recorded audio in digital format. A touch button triggers the start and stop of recording. When switched in the playing mode, the different microprocessors from the different recorders must detect the order in which they are arranged, to identify the first one, as well as the order of the whole sequence, to send their respective data to the player. Each recorder is power supplied by a small rechargeable battery that charges automatically when connected to the player.

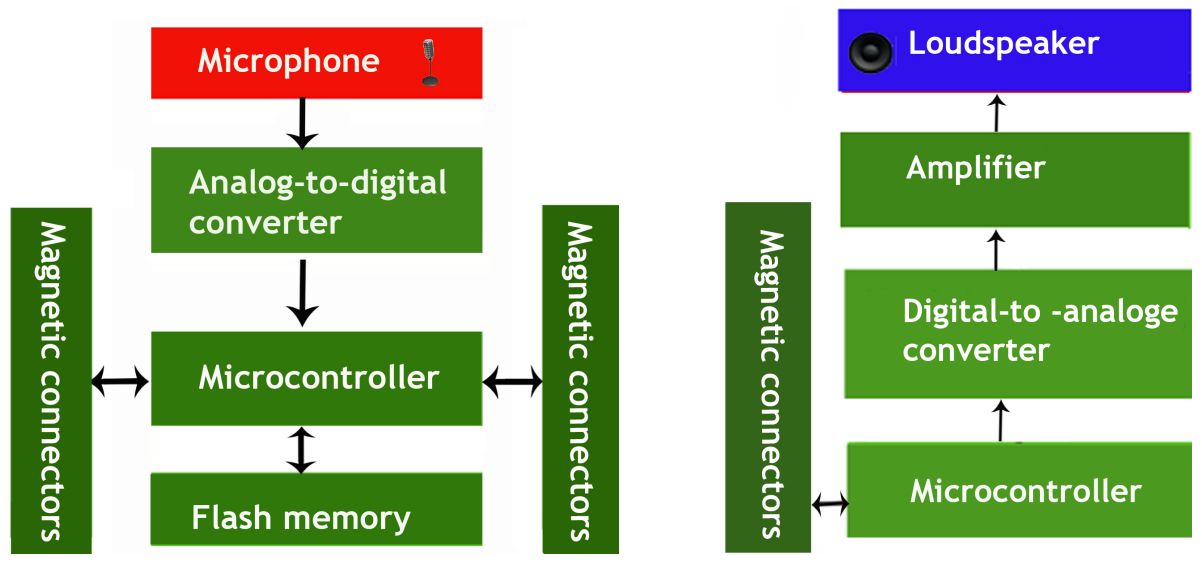

Fig. 3. Diagram of a recorder block (left) and of a player -block (right)

The player has a microcontroller that receives the audio in digital format from the recorders, and then sends it to a loudspeaker through a digital-to-analog converter and a sound amplifier (Fig.3 right). The audio data is requested from the recorders as needed. The player's power is supplied by a set of non-rechargeable batteries, capable 
of supplying the entire system. The communication protocol is serial-based allowing pieces to connect to each other, through magnetic connectors. Among a subgroup of pieces there is always just a single masterpiece, the remaining others are slaves, the master's function is to retain the information about the relative order by which the pieces are connected. When the player is added, it automatically becomes the master and manages the entire row, requesting the necessary data from the corresponding recorders. The blocks can be arranged and rearranged so that children can playful engage in trying out different possibilities.

\section{Conclusions}

Teachers can use t-words to propose a wide range of activities that can help children to foster early literacy and language development through improvement of phonological sensitiveness, and creatively engaging in storytelling. They may create rhymes that match a certain word; record words or sentences about a certain subject, or create a narrative. Additionally the design of the interface encourages children's collaboration, as they have to handle the best way of bringing the different blocks together.

Acknowledgements. This work is funded by FEDER through the Operational Competitiveness Factors Program - COMPETE and by National Funds through the FCT - Portuguese Foundation for the Science and the Technology within the Project: PTDC/CPE-CED /110417/2009, and the Doctoral Grant: SFRH /BD / 62531 / 2009.

\section{References}

1. Ananny, M.: Telling Tales: A new toy for encouraging written literacy through oral storytelling. In: Society for Research in Child Development, Minneapolis (2001)

2. Arduino official web site, http: / / www . arduino.cc/

3. Clay, M.M.: Emergent Reading Behavior. Unpublished doctoral dissertation, University of Auckland, New Zeland (1996)

4. Hunter, S., Kalanithi, J., Merrill, D.: Make a Riddle and TeleStory: Designing Children's Applications for the Siftables Platform. In: Proceedings of the 9th IDC International Conference on Interaction Design and Children, pp. 206-209. ACM, New York (2010)

5. Raffle, H., Vaucelle, C., Wang, R., Ishii, H.: Jabberstamp: embedding sound and voice in traditional drawings. In: Proceedings of the 6th IDC International Conference on Interaction Design and Children, pp. 137-144. ACM, New York (2007)

6. Sylla, C., Gonçalves, S., Brito, P., Branco, P., Coutinho, C.: t-books - Merging Traditional Storybooks With Electronics. In: Proceedings of the 11th IDC International Conference on Interaction Design and Children, pp. 323-326. ACM, New York (2012)

7. Whitehurst, G.J., Lonigan, C.J.: Child Development and Emergent Literacy. Child Development 69(3), 848-872 (1998) 